\title{
Towards on the Mediators in Collagen Replacement: Effect of the Active Molecules into a Polymeric Biomaterial
}

\author{
Anderson Ricardo Ingracio, Natália Fontana Nicoletti², Fernanda Trindade Gonzalez Dias ${ }^{3}$, \\ Manuela Peletti-Figueiró ${ }^{2 *}$, Otávio Bianchi ${ }^{1,3}$, Asdrubal Falavigna ${ }^{1,2}$ \\ ${ }^{1}$ Health Sciences Graduate Program, Universidade de Caxias do Sul (UCS), Caxias do Sul, RS, Brazil \\ ${ }^{2}$ Cell Therapy Laboratory (LATEC), Universidade de Caxias do Sul (UCS), Caxias do Sul, RS, Brazil \\ ${ }^{3}$ Materials Science Graduate Program (PGMAT), Universidade de Caxias do Sul (UCS), Caxias do Sul, RS, Brazil \\ Email: *manu.peletti@gmail.com
}

How to cite this paper: Ingracio, A.R., Nicoletti, N.F., Dias, F.T.G., Peletti-Figueiró, M., Bianchi, O. and Falavigna, A. (2021) Towards on the Mediators in Collagen Replacement: Effect of the Active Molecules into a Polymeric Biomaterial. Journal of Biosciences and Medicines, 9, 26-42. https://doi.org/10.4236/jbm.2021.96003

Received: April 24, 2021

Accepted: June 1, 2021

Published: June 4, 2021

Copyright $\odot 2021$ by author(s) and Scientific Research Publishing Inc. This work is licensed under the Creative Commons Attribution International License (CC BY 4.0).

http://creativecommons.org/licenses/by/4.0/

(c) (i) Open Access

\begin{abstract}
Modulating healing factors could avoid or minimize some possible pathological processes in collagen deposition. The present study was aimed to evaluated the role of active biomolecules such as PDGF-BB and PRP loaded or not into polymeric biomaterial to seek potential mediators in types I and III collagen deposition and epithelization. The healing phases were investigated by using an in vivo full-thickness wound rat model. At zero, $3^{\text {rd }}, 7^{\text {th }}$ and $14^{\text {th }}$ days after the experimental model, the size of the wound areas was photographed. The nanofibrous materials were biocompatible and did not cause any local adverse reaction and/or inflammation. On day 14 the wounds had healed almost $100 \%$ with better signs of healing, however there was no obvious difference in the wound contraction rates. At the end of 14 days, samples from the center of the lesion were collected when histological features and immunopositivity for collagen I and III expressions were assessed. There was no significant difference in the epithelization among the groups. Wounds treated with PRP and with PA-6/SOMA plus PDGF-BB had significantly lower amounts of type III collagen. The amounts of type I collagen did not have a statistically different deposition among the experimental groups. The association of PDGF-BB with PA-6/SOMA emerges as an alternative for topical application to unfavorable anatomical sites, suggesting that these associations may have a positive modulation on the process of accelerated healing remodeling.
\end{abstract}

\section{Keywords}

Collagen, Fibrosis, Platelet-Rich Plasma, Polyamide- 6 and Biomaterial 


\section{Introduction}

Tissue healing is a dynamic and coordinated sequence of cellular events common to all histological lines to restore their anatomical structure, appearance and functionality [1] [2] [3]. Unlike regeneration, unusual in mature human tissue, healing evokes tissue changes that distinguish it from the original histological tissue features [4]. The smaller the difference between the tissue characteristics and those present after healing, the lower the impact on its functionality [1] [2] [4].

Regardless of damaged tissue, the extracellular matrix (ECM) has a decisive role and constitutes the anatomical framework that confers resistance [2] [5]. This matrix is characterized by an abundant growth factors (GF) environment that modulates angiogenesis and tissue development [1] [4] [6]. Long and dense collagen fibers play an essential role in tissue resistance and in the interaction between resident cells, soluble mediators and ECM molecules [3] [4] [6]. In this sense, collagen synthesis is a pivotal event in the physiological cicatricial process, since deficient deposition and/or altered lysis can disrupt the course of wound healing [2] [5]. Increased collagen deposition, associated with an alteration in histological architecture, leads to the progression of the pathological process known as fibrosis [5]. Fibrosis is an expected event in invasive procedures, but its intensity can induce a retraction and dysfunction of anatomical structures [7] [8]. A classical phenomenon is the symptomatic retraction of spinal epidural fibrosis that triggers chronic neuropathies with high refractoriness to pharmacological or surgical treatment [7] [9] [10].

To improve the application of modulating factors in specific anatomical sites, it is necessary to study the ideal method for local delivery of biomolecules. One approach includes direct deposition of the biomolecule at the wound site, while another considers the use of a biomaterial as a carrier vehicle for local release. It is known that the administration of these biological agents may lead to some undesirable events, such as rapid degradation by proteases in the wound environment [11]. GF that are currently approved by the US Food and Drug Administration (FDA) has been used as some form of delivery system [12]. Controversially, platelet-rich plasma (PRP) is commonly used by direct application into wound site and there is no consensus about its performance as a specific modulator in the healing phases [13].

The advantages of using biomaterials as delivery vehicles are that biomolecules can be protected from endocytosis and have structural support on which the host cells can migrate to facilitate tissue regeneration. A nanofibrillar scaffold based on a synthetic polymer was chosen to be tested as a system for topical biomolecule delivery in this study. Polyamide-6 (PA-6) is a FDA-approved polymer for human use in surgical sutures, especially for its structural similarity with proteins [14]. When chemically modified with maleinized soybean oil (SOMA), PA-6 becomes very tenacious, which favors biomaterial handling and cellular morphogenesis [15]. PA-6/SOMA scaffolds showed superior biocompa- 
tibility and an intriguing 3D architecture, as attested in a previous work by our group [16]. PA-6/SOMA biomaterial also exhibited a covalent-binding interaction with PDGF-BB, which may contribute to the controlled-release of the biomolecules.

The present study aimed to evaluate collagen deposition and epithelialization during the healing phase, using an in vivo model to verify the role of active biomolecules such as PDGF-BB and PRP loaded or not with PA-6/SOMA. This work focused on the influence of the strategy chosen for biomolecule administration in order to evaluate possible modulations in contraction rates and collagen type III to type I replacement in full thickness wounds to handle the fibrosis response after tissue injury.

\section{Materials and Methods}

\subsection{Animal Model and Ethical Approvals}

Male Wistar rats (200 - $300 \mathrm{~g}$; $\mathrm{N}=36$ ), were obtained from the Federal University of Rio Grande do Sul (UFRGS). During the set of experiments, the animals were kept in the Laboratory of Animal Physiology and Experimentation at the University of Caxias do Sul (UCS). All the experimental procedures following the Brazilian Guideline for Care and Use of Animals for Scientific and Educational Purposes (MCTI, CONCEA, DBCA, 2013) and the recommendations for laboratory animal care and ethical standards for experimental animal testing in the National Institute of Health (NIH) Laboratory Use and Care Guide. All the experimental protocols were approved by the local Animal Ethics Committee (CEUA-UCS; protocol number: 017/2016).

The animals were housed in cages under conditions of optimum light, temperature and humidity $\left(12 \mathrm{~h}\right.$ light-dark cycle, $22^{\circ} \mathrm{C} \pm 1{ }^{\circ} \mathrm{C}$, under $60 \%$ to $80 \%$ humidity). Animals received pelleted rodent feed and filtered water ad libitum. No experimental procedure was performed in the room where the animals were maintained, to avoid any type of behavioral stress. In the postoperative period, animals were maintained on a thermal mattress until recovery and housed in individual cages. Analgesia was performed by administering tramadol (5 to 7.5 $\mathrm{mg} / \mathrm{kg}$, s.c.) every 12 hours for 3 to 5 days as individually needed. The animals were euthanized by isoflurane overdose fourteen days after the procedure, when the skin wound was completed.

\subsection{Study Design: Full-Thickness in Vivo Wound Model}

The animals were anesthetized with ketamine hydrochloride $(80 \mathrm{mg} / \mathrm{kg})$ mixed with xylazine $(10 \mathrm{mg} / \mathrm{kg})$ intraperitoneally. After trichotomy, the animals were positioned in ventral decubitus and the areas of dorsal lesions were measured and demarcated. Concerning the dorsal midline, each animal was subjected to two full-thickness incisional circular cutaneous resections, $1 \mathrm{~cm}$ in diameter using a cutaneous punch until the dorsal muscular fascia was exposed. There was a $1.5 \mathrm{~cm}$ separation between the lesion areas to prevent contact and possible 
extravasation of the active biomolecule. The procedures were carried out by the same operator to avoid technique variations. A different experimenter randomly allocated the animals into the different groups.

Animals were randomly divided into the following experimental groups $(\mathrm{N}=$ 6 per group): 1) control/PFGF-BB group; 2) PA-6/SOMA + PDGF-BB group; 3) PA-6/SOMA + PRP group. The control/PDGF-BB group received no application to the left lesion (L) and received the application of PDGF-BB only to the right lesion (R). In the $P A-6 / S O M A+P D G F-B B$ group, the polyamide nanomembrane alone was placed inside lesion $\mathrm{L}$, while the biomaterial loaded with PDGF-BB was kept in lesion R. The $P A-6 / S O M A+P R P$ group received PRP in lesion $\mathrm{L}$ and PA-6/SOMA plus PRP in lesion R (Figure 1). No membranes applied to on the wounds were sutured to the skin. The wound sites were observed macroscopically within a 14-day follow-up.

\subsection{Isolated or Associated Use of Platelet-Rich Plasma (PRP) in the Lesion}

Whole blood $(6 \mathrm{~mL})$ was collected by cardiac puncture from donor animals by using an anticoagulant in a proportion of $20 \%$ of the total blood volume. The blood was centrifuged for 10 minutes at $1000 \mathrm{rpm}$ to separate the plasma and hemocyte fraction. The plasma fraction and the mist zone were transferred to a

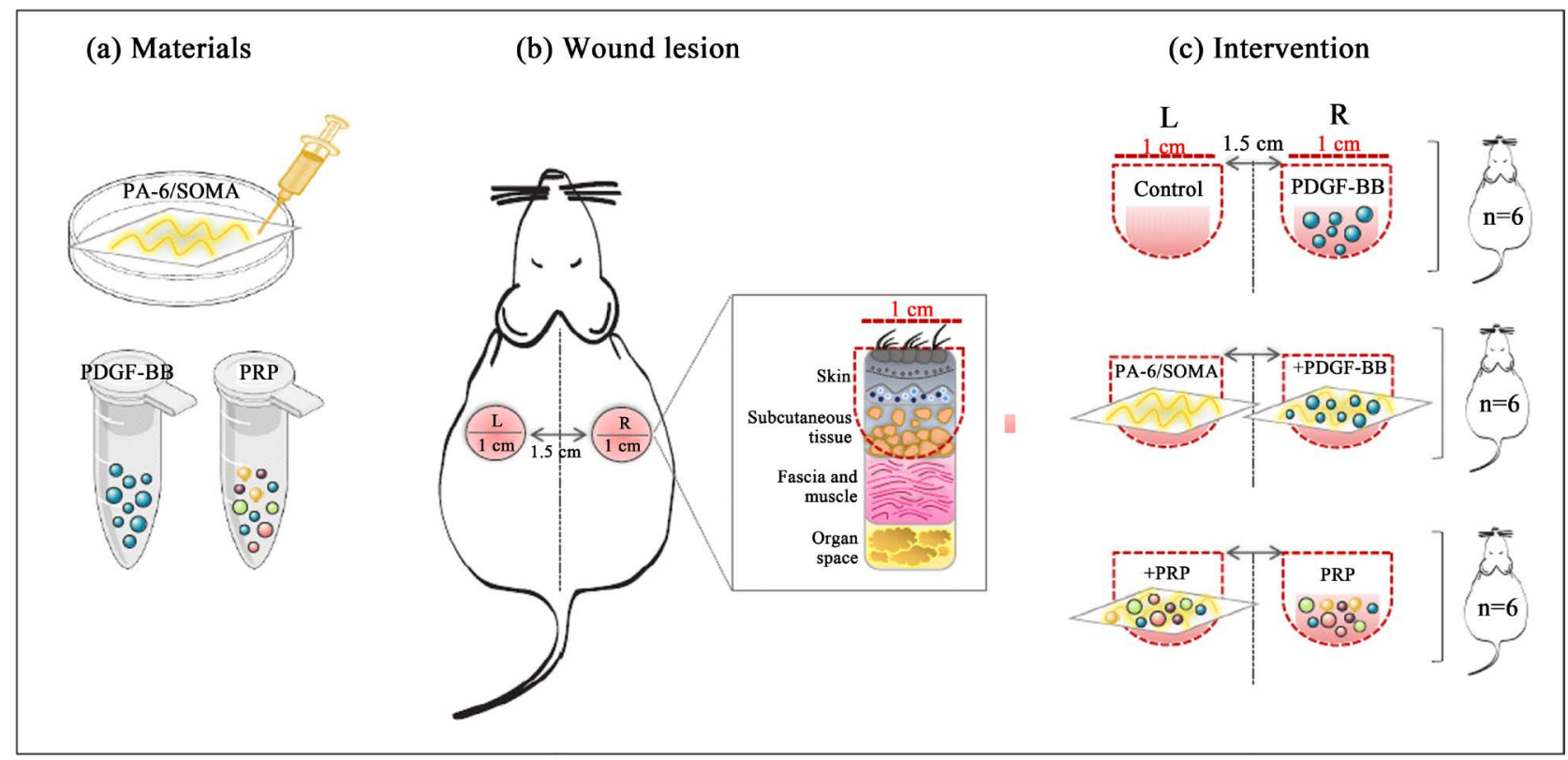

Figure 1. Schematic study design of the experimental groups submitted to full-thickness in vivo wound model. (a) Materials applied on the wound site. PA-6/SOMA, maleinized soybean modified polyamide-6; PDGF-BB, platelet derived growth factor-BB and PRP, platelet-rich plasm. (b) Wound lesion based on full-thickness wound when each animal was subjected to two incisional circular cutaneous resections, $1 \mathrm{~cm}$ in diameter using a cutaneous punch until the dorsal muscular fascia was exposed. (c) Animals were randomly divided into the following experimental groups $(\mathrm{N}=6$ per group): The control/PFGF-BB group received no application to the left lesion (L) and received application of growth factor PDGF-BB only to the right lesion (R). In the $P A-6 / S O M A+P D G F-B B$ group, the biomaterial enriched with maleinized soybean oil was applied to lesion left (L) and the same biomaterial loaded with PDGF-BB to lesion right (R). The $P A-6 / S O M A+P R P$ group received PRP in lesion left (L) and biomaterial PA-6/SOMA plus PRP in lesion right $(\mathrm{R})$. 
new tube and centrifuged for 10 minutes at $1600 \mathrm{rpm}$. Three distinct layers were obtained and the intermediate layer, designated as PRP, was collected. The mean number of platelets in whole blood of PRP-donor rats was $89.6 \times 10^{4}$ platelets $/ \mu \mathrm{L}$. After PRP preparation, the platelet count was $340 \times 10^{4} / \mu \mathrm{L}$ producing a 3.8-fold increase in concentration compared to native plasma. The number of platelets of each PRP fraction was counted by using a hemocytometer to reach a concentration of 10,000 platelets/ $\mu \mathrm{L}$. Next, $20 \mu \mathrm{L}$ of the fresh PRP was used on cutaneous wounds topically, injected into the center of the lesion, or incorporated into PA-6/SOMA nanomembrane.

\subsection{Isolated or Associated Use of Recombinant Human PDGF-BB in the Lesion}

Recombinant human platelet-derived growth factor-BB (PDGF-BB) (Pepro Tech Inc., Rocky Hill, NJ) was reconstituted in $0.1 \%$ BSA and diluted with ultra-pure water to achieve a concentration of $10 \mu \mathrm{g} / \mathrm{mL}$ and stored at $-80^{\circ} \mathrm{C}$. PDGF-BB $(100 \mathrm{ng})$ in solution was directly injected into the center of the lesion or added to PA-6/SOMA nanomembrane and incubated for 45 minutes at $37^{\circ} \mathrm{C}$.

\subsection{PA-6/SOMA Nanomembrane as a Carrier Vehicle}

A chemically modified PA-6 (PA-6/SOMA) was tested as a carrier agent of the biological factor (PDGF-BB or PRP) to assess its benefit for topical delivery, as well as wound healing. PA-6/SOMA nanomembrane was produced by electrospinning technology, from polymeric solutions under the experimental conditions: $0.1 \mathrm{ml} / \mathrm{h}$ feeding rate, $15 \mathrm{~cm}$ syringe-collector distance and $25 \mathrm{kV}$ applied voltage. The electrospinning process and the structure of this nanomembrane are represented in Figure S1 (see in supplementary files). The PA-6/SOMA mats were composed of uniform nanofibers with the diameter $(250.0 \pm 9.0 \mathrm{~nm})$ within range of extracellular matrix collagen fibers (ECM). Dias et al. (2017) [16] thoroughly discussed the properties of PA-6/SOMA scaffold.

\subsection{Morphometric Analysis of the Lesion Area}

At zero (lesion time), $3^{\text {rd }}, 7^{\text {th }}$ and $14^{\text {th }}$ days after the experimental model, the size of the wound areas was photographed on a perpendicular by means of a digital camera (Fujifilm Finepix HS10) with $35 \mathrm{~mm}$ lens aperture and $27.5 \mathrm{~cm}$ distance between lens and bench under the same conditions each time. The analysis of the non-epithelial lesions in the pictures was marked and the number of pixels within the bordered area was measured to calculate the wound area by using Image J-Pro Plus 4.0 software (Media Cybernetics L.P). The wound area contraction rate $(\mathrm{C})$ was calculated based on the measured area on day zero (A0; postoperative) and the area reduction rate on the 14th day (A14), through the following expression: $\mathrm{C}=\mathrm{A} 0-\mathrm{A} 14$. The rate of cicatricial contraction (TC) was determined from the previous results by means of $\mathrm{TC}=\mathrm{C} \times 100 / \mathrm{A} 0$. 


\subsection{Histological Analysis and Staining of Hematoxylin \& Eosin}

After the 14-day follow-up, samples from the center of the lesion were collected and immediately fixed in $10 \%$ buffered formalin $(\mathrm{pH}$ 7.0) for further processing and analysis. Serial sections of $5 \mu \mathrm{m}$ were stained with hematoxylin and eosin (HE). Histological observation and measurements of the presence or absence of reepithelialization were performed under a optical microscope (Leica DM2500, Leica Microsystems, Bannockburn, IL, U.S.A.) equipped with an automated Leica digital camera system (Leica DMC2900, Leica Microsystems) and an image manager software (LASV4.4, Leica Microsystems). The analyses were performed by an experimenter blinded to the experimental groups (images represented in Figure S2-see in supplementary files).

\subsection{Immunohistochemistry (Evalution of Collagen I and III)}

Immunopositivity for collagen I and III expression were assessed on paraffin tissue sections $(3-\mu \mathrm{m}$ thick) by using polyclonal rabbit anti-collagen I (1:100, Cat \#ab34710, abcam, Cambridge, United Kingdom), and polyclonal rabbit anti-collagen III (1:50, \#ab7778, abcam, Cambridge, United Kingdom), according to the procedures previously described [17]. The samples were treated with an amplifier (HiDef Detection Amplifier Mouse \& Rabbit-Cell Marque) followed by the detection polymer (HiDef Detection HRP Polymer Detector-Cell Marque). The materials were stained with DAB (Diaminobenzidine-Cell Marque) and counterstained with Harris Hematoxylin [17]. Images were examined with a Leica DM2500 optical microscope (Leica Microsystems, Bannockburn, IL, U.S.A.). Four images of each wound lesion were captured in $\times 200$ magnification. To quantify the immunopositive collagen I and III, digitized RGB images were analyzed using Image NIH Image J 1.36b Software (NIH, Bethesda, MD, USA). For this purpose, a specific macro was created for each sample to quantify the collagen type immunopositivity based on pixel color. The dark-to-medium brown regions in the sample were selected and used as positive immunolabeling. The software generated a macro, which allowed determining the optical density of the positive regions.

\subsection{Statistical Analyses}

Data from experimental results were statistically processed using program $\mathrm{R}$ version 3.4.4., considering $5 \%$ significance $(\alpha)$ and $90 \%$ statistical power. A difference of standardized means greater than or equal to 1.5 standard deviation units between groups $(E / S \geq 1.5)$ was detected. The morphometric results between groups were compared through a linear mixed model for repeated measures with localization of Tukey's post-hoc test. The variance of measures compared the concentration of collagen (histomorphometric analysis).

\section{Results}

\subsection{Clinical Aspects and Macroscopic Evaluation of the Wound}

The cutaneous wound was macroscopically evaluated in vivo during 14 days. 
The wound site presented a homogenous surface without signs of granulation tissue exposure and developed a crust adhered to the injury site from the $3^{\text {rd }}$-day post procedure. The wound edges retracted regularly in all animals and at 14 days the wounds were almost completely healed. The response of the wound site denoted that the presence of the nanofibrous carrier did not cause any local adverse reaction and/or inflammation, as did also, the direct application of PDFG-BB or PRP into the center of the wound site (Figure 2(a)). In the wounds in which the biomaterial was applied, also there was the formation of a neat crust on and around it, leading to the creation of an interfacial tissue that was later incorporated by the newly tissue formed. The biomaterials remained fixed at the wound site the entire time and did not need sutures to keep them in position.

\subsection{Wound Closure}

Photographic images of the wound healing progress were captured at four points in time (0, 3, 7 and 14 days) and are summarized in Figure 2(a). There was no noticeable difference in the speed of wound contraction among the animals treated by PDGF-BB or PRP loaded or not in PA-6/SOMA at the specified times. A marked improvement was seen after 7 days post-procedure when the injuries became covered by a solid, intact and dark brown scab. Thenceforward, the wounds started to slough off leaving behind minimal crust remnants in predominantly

(b)

(a)

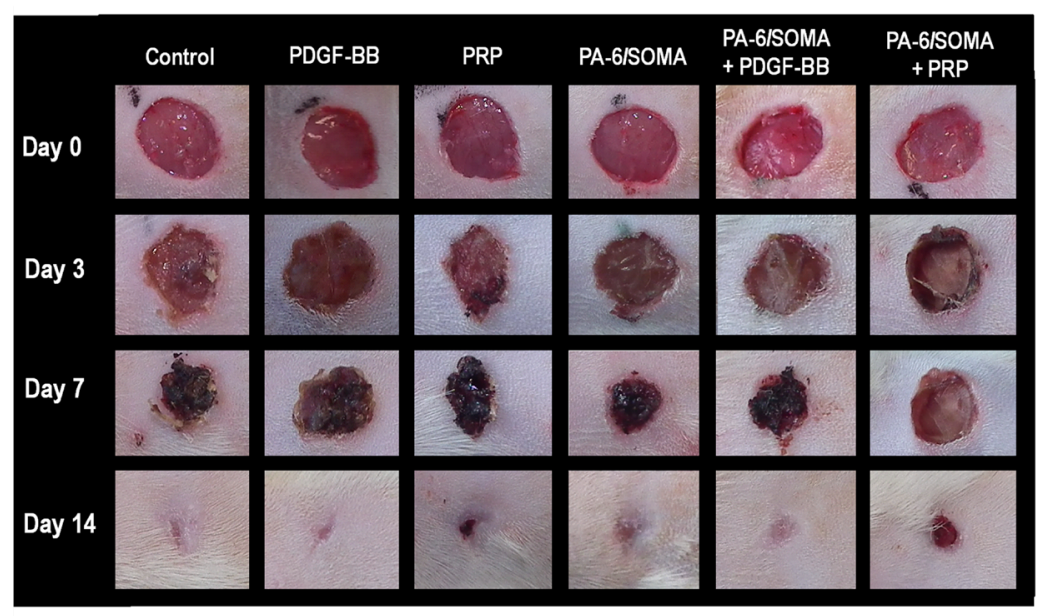

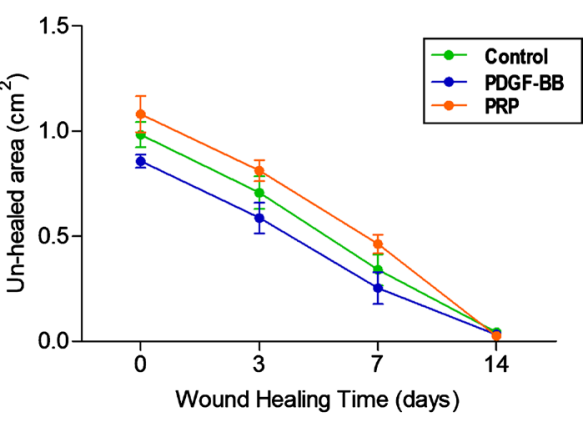

(c)

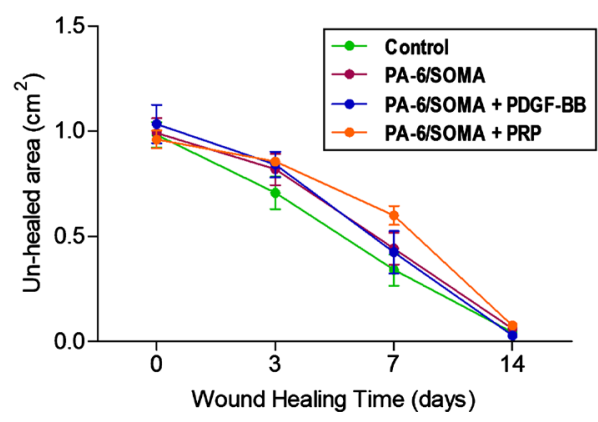

Figure 2. Time course of wound healing. (a) Representative pictures of wound lesions at different stages of the wound healing process at $0,3,7$ and 14 days; ((b), (c)) The points represent the average wound size ( $\mathrm{n}=6$ animals) over the days of the experiment for each treatment regimen. Data were presented as means $\pm S D(n=6)$ and the morphometric analysis between the groups during the observation period was determined by ANOVA with Tukey's post hoc test. PA-6/SOMA, maleinized soybean modified polyamide-6; PDGF-BB, platelet derived growth factor-BB and PRP, platelet-rich plasm. 
epithelized wounds that become progressively smaller. On day 14 the wounds were almost closed, achieving nearly $100 \%$ of closure with better a sign of healing (Figure 2(b) \& Figure 2(c); Table 1). It is important to emphasize that the physical strength generated by the biomaterial application on the wound site did not delay wound contraction.

\subsection{Histological Findings}

Epithelization is an essential component of wound healing used as a defining parameter of successful wound closure. The epithelized areas at the wound were easy to see from the images of HE-stained sections. There was no significant difference in the epithelialization among the groups, suggesting that they did not accelerate the epithelization of full-thickness skin defect wounds in a 14-day follow-up period (Table 1). However, the physical presence of the biomaterial did not inhibit the epithelization rates too.

\subsection{Collagen I and III}

Deposition of type I and type III collagen was measured at 14 days follow-up. This event corresponds to the phase of cicatricial remodeling in rats, initiated about 10 days after the injury. In this phase, there should be the exchange of collagens, with the replacement of collagen type III by collagen type I. In absolute numbers, it was observed differences in the collagen deposition of the groups in relation to the control. A tendency to smaller amplitude of difference between the two types of collagen was observed in the groups treated with PA-6/SOMA. Wounds treated with PRP and PDGF-BB-loaded PA-6/SOMA had lower amounts of type III collagen. The amounts of type I collagen did not have a statistically different deposition between the groups compared to the control group, like is presented in Figure 3. The difference in the amount of type III collagen is

Table 1. Measurements of wound area contraction, epidermal thickness and collagen type III and I deposition.

\begin{tabular}{|c|c|c|c|c|c|c|c|c|c|}
\hline Group & $\begin{array}{r}\text { Wound } \\
(\mathrm{cr} \\
\text { Day } 0\end{array}$ & $\begin{array}{l}\text { Contraction } \\
\left.\mathrm{a}^{2} \pm \mathrm{SD}\right) \\
\text { Day } 14(\%)\end{array}$ & & $\begin{array}{l}\text { Epidermal } \\
\text { Thickness } \\
(\mu \mathrm{m} \pm \mathrm{SD})\end{array}$ & $\mathrm{p}\left({ }^{*}\right)$ & $\begin{array}{l}\text { Collagen III } \\
(\mathrm{OD} \pm \mathrm{SD})\end{array}$ & $\mathrm{p}\left({ }^{*}\right)$ & $\begin{array}{l}\text { Collagen I } \\
(\mathrm{OD} \pm \mathrm{SD})\end{array}$ & $\mathrm{p}\left({ }^{*}\right)$ \\
\hline Control & $0.983 \pm 0.06$ & $0.043 \pm 0.007$ & 95.9 & $83.71 \pm 54.14$ & ref. & $128,594.28 \pm 13,155.05$ & ref. & $64,132.17 \pm 30,594.86$ & ref. \\
\hline PDGF-BB & $0.857 \pm 0.031$ & $0.032 \pm 0.003$ & 96.2 & $106.32 \pm 30.08$ & 1.00 & $113,938.06 \pm 37,347.56$ & 1.00 & $67,298.89 \pm 34,439.19$ & 1.00 \\
\hline PRP & $1.080 \pm 0.086$ & $0.026 \pm 0.003$ & 97.5 & $108.11 \pm 26.30$ & 1.00 & $78,460.39 \pm 29,341.07$ & $0.003(*)$ & $26,434.11 \pm 14,417.77$ & 0.17 \\
\hline PA-6/SOMA & $0.991 \pm 0.071$ & $0.060 \pm 0.010$ & 93.6 & $121.91 \pm 27.82$ & 1.00 & $107,178.44 \pm 29,728.79$ & 1.00 & $48,067.50 \pm 22,569.19$ & 1.00 \\
\hline $\begin{array}{l}\text { PA-6/SOMA } \\
+ \text { PDGF-BB }\end{array}$ & $1.034 \pm 0.090$ & $0.029 \pm 0.004$ & 97.3 & $109.18 \pm 66.52$ & 1.00 & $72,086.56 \pm 21,666.83$ & $<0.001\left(^{\star}\right)$ & $49,217.39 \pm 9169.89$ & 1.00 \\
\hline $\begin{array}{c}\text { PA-6/SOMA } \\
\text { + PRP }\end{array}$ & $0.960 \pm 0.043$ & $0.075 \pm 0.020$ & 92.1 & $107.39 \pm 36.95$ & 1.00 & $97,463.61 \pm 32,744.78$ & 0.67 & $50,767.94 \pm 27,534.09$ & 1.00 \\
\hline
\end{tabular}

OD: Optical density; PA-6/SOMA: maleinized soybean modified polyamide-6; PDGF-BB: platelet derived growth factor-BB; PRP: platelet-rich plasm. The measurements of wound area contraction rate $(\mathrm{C})$ was calculated based on day zero (A0; postoperative) and the reduction area rate obtained on the day 14 (A14), calculated by the following expression: $\mathrm{C}=\mathrm{A} 0-\mathrm{A} 14$. The rate of cicatricial contraction (TC) was obtained from the previous results by means of TC $=\mathrm{C} \times 100 / \mathrm{A} 0 . \mathrm{P}<0.05\left(^{*}\right)$ : comparison to the respective control. Data were presented as means \pm Standard Deviation $(\mathrm{SD})$. 
(a)

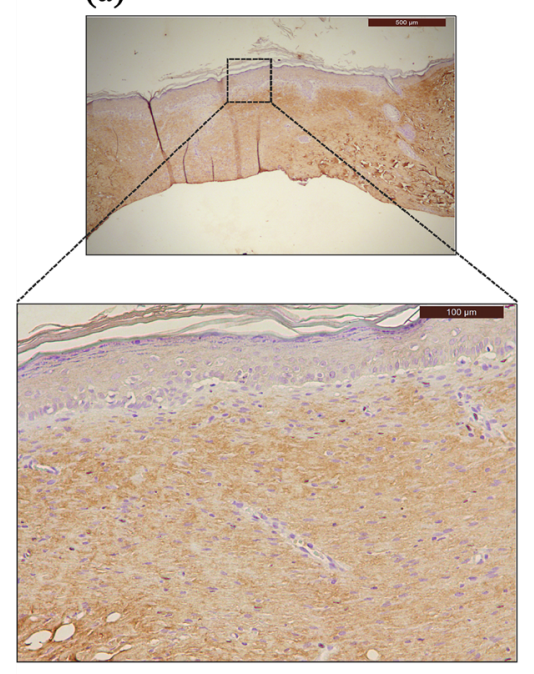

(c) (b)

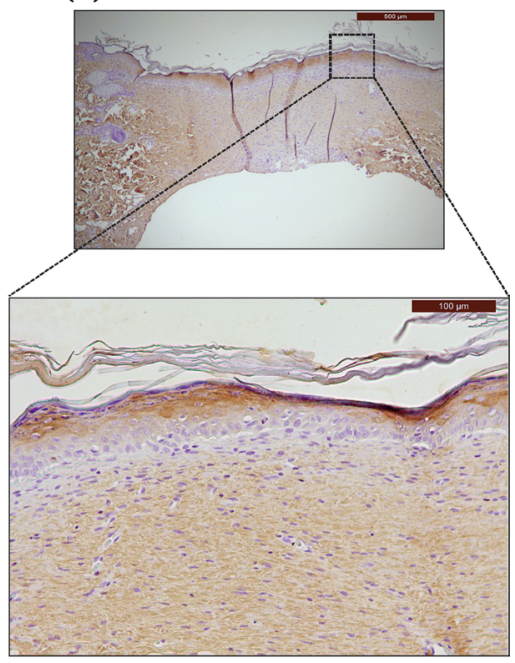

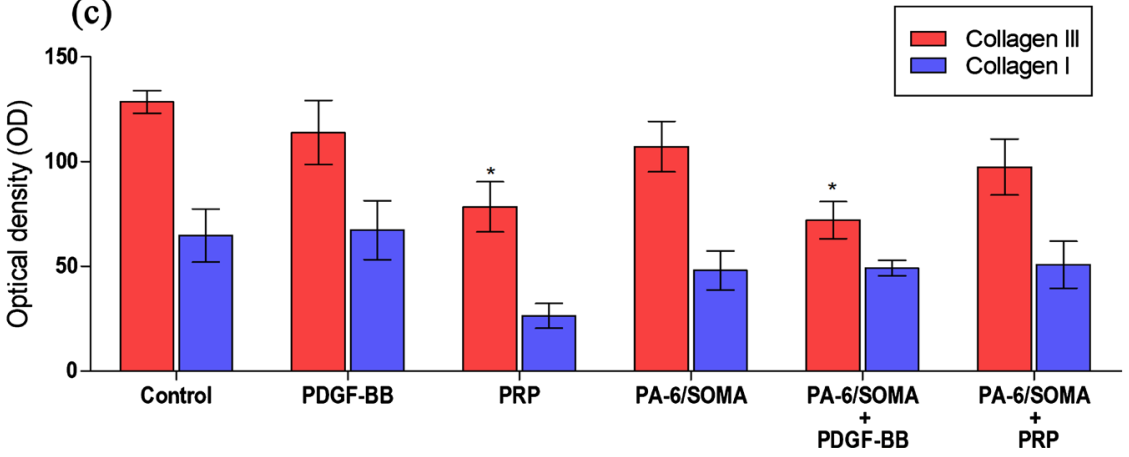

Figure 3. Immunohistochemistry analysis on the type III and type I collagen deposition. ((a), (b)) Representative images of immunohistochemistry analysis for type III and type I collagen density in paraffin-embedded tissues. Scale bar $=50 \mu \mathrm{m} ; 100 \mu \mathrm{m}$; (c) Quantification of type III and type I collagen deposition by Image J software. Each column represents the mean $\pm S D(n=6) .{ }^{*} p<0.05$ for comparison versus control group determined by ANOVA. PA-6/SOMA, maleinized soybean modified polyamide-6; PDGF-BB, platelet derived growth factor-BB and PRP, platelet-rich plasm.

especially crucial in the PDGF-BB-loaded PA-6/SOMA. The result found for isolated PRP was not replicable when this biological factor was associated with PA-6/SOMA. A smaller amount of type III collagen, without loss of type I collagen deposition, is the primary finding of the cicatricial remodeling process.

\section{Discussion}

Every tissue disruption with consecutive loss of organ functions might be described as a wound and it is necessary to be able to rely on clinically efficient healing whenever a patient receives surgical intervention. If the surgical wound healing fails to progress in a timely and orderly fashion to repair the injured tissue it leads to an unspecified form of closure and causes fibrosis and scar formation [1].

The present work used a biomaterial consisting of polymeric nanofibers as a vehicle to improve the local application of PRP and PDGF-BB. Concerning the 
topical application of PA-6/SOMA to the wounds, two topics are especially considered: if the biomaterial was effective in delivering the biological compounds locally and if its their presence per se did not negatively alter the process. According to the results, both PDGF-BB and PRP applied alone or in association with PA-6/SOMA presented the same healing rates. Natural and synthetic polymers have been used for biomolecule delivery in in vivo animal wound healing studies. Compared with cellular scaffolds, these polymeric matrices exhibit better ECM formation although no significant changes in healing rates [18] [19] [20]. A controlled release of bFGF and EGF biomolecules incorporated in synthetic polymers promoted faster wound closure in 14 days [21] [22]. Of note, the physical strength generated by the PA- 6 based biomaterial application on the wound site did not undermine the healing process, which confirms the potential of PA-6/SOMA being applied as a vehicle for topical delivery. This feature is a pivotal point considered to create polymeric textiles for drug delivery aiming at tissue repair and healing.

Satisfactory wound contraction was achieved by using natural products and cell sources but without being hampered by a scaffold, when its physical presence might be precluded wound contraction [23] [24]. In this sense, the same animal studies also had demonstrated accelerated healing when the wound bed was supplied by GF or PRP. Incorporation of PDGF-BB or PRP in PA-6/SOMA biomaterial would be an alternative for topical application at unfavorable anatomical sites. Also, a carrier that supports biomolecules becomes a protective factor, preventing simultaneously their rapid degradation by proteases and diffusion in the wound environment.

PDGF presents wound healing activities in both bone and soft tissue. Currently, the only FDA approved GF is the human recombinant PDGF-BB [25]. The commercially available formulations of PDGF-BB use a high concentration $(100 \mu \mathrm{g} / \mathrm{ml})$ in order to heal wounds effectively. This high concentration likely leads to elevated systemic levels of GF that may drive adverse reactions. Therefore, wound dressings that improve the efficacy of PDGF-BB have a great potential in postsurgical wound complications. PRP is defined as a higher concentrate of platelets when compared to whole blood levels, presenting a higher density of GF [11] [26] [27]. Platelet function is widely known and established in hemostasis [4] [10] [13], the principal standpoint of the perfect healing. The platelets concentration necessary to be recognized as PRP has not been established and a platelet level 3 to 4 times that of whole blood is well accepted [11] [13] [26] [27]. In our study, it was followed previous recommendations for PRP manufacture and the methodology applied was achieved, with a supply of autologous concentrate rich in GF.

The soybean oil derivative chains of PA-6/SOMA are constituted by fatty acids and tocopherols, related to anti-inflammatory and epithelizing properties which makes it a possible wound-healing accelerator. At first, the PDGF-BB and PRP used alone or in combination with this biomaterial were not able to mod- 
ulate the epithelization on the healing process. Although epithelization is an essential component of wound healing used as a defining parameter of its success, the difference seen between samples can be understood by the amplitude that the correct measurement of its physiological characteristics, considering its papillary projections, needs to be contemplate.

PA-6/SOMA did not degrade by physiological process of cells and spread of tissue fluids over the 14 days of follow-up. In time, the body breaks down the biomaterial and only a newly formed skin layer should remain. Some intracellular fragments of a dermal polymeric scaffold were detected by electronic microscopy in five patients with burn injury one-year post-treatment [28]. The presence of these fragments indicated that cells could phagocytize the biomaterial and that it did not cause any adverse reaction in the organism. This justifies the advantage of a slow-degradable biomaterial, in addition to its mechanical support that guarantees the tissue ingrowth.

Closely related to proper ECM deposition is the degree of wound contraction. During the wound remodeling process, there is a replacement of collagen fibers, such that quality of ECM cicatricial process results from the balance between its neoformation and lysis [1] [2] [5] [6]. Collagen I must gradually replace collagen III to increase mechanical stability of the tissue and resist over-contraction by myofibroblasts, which is associated with pathological scarring [29].

Increasing the rate of wound closure is not necessarily an improvement in itself. PDGF-BB-loaded PA-6/SOMA showed positive modulation on tissue remodeling by collagen replacement. This event is considered a key on healing acceleration in different histological lines [18] [21] [30]. The deposition of dermal collagen, even as an isolated measure, can be correlated with the activity of pathological processes in parallel with the marker function proposed in the tissue repair events [29]. When not part of the healing process, its modulation may not interfere with clinical outcome.

Collagen III levels are generally up-regulated in the early phase of wound healing and mechanically poor tissues [31]. The down-regulation of collagen III at 14 days post-injury in rats treated with PDGF-BB-loaded PA-6/SOMA indicates good mechanical tissue properties. Furthermore, the increased density of type I collagen would probably continue beyond 14 days if the animal model follow-up had been extended, suggesting rapid and continuous collagen replacement from type III to type I. A previous animal study with wounds topically treated with lavender oil demonstrated that suppression of type III collagen in the wound area enhanced myofibroblast expression and differentiation to promote wound contraction [24] [32]. Polymeric biomaterial coated with bFGF allowed the controlled release of the biomolecule and led to a significant enhancement of collagen I density in addition to better elastin and collagen structure in diabetic rats submitted to a full-thickness wound [21] [22]. Using the same animal model, a recent study took an advanced approach to enable controlled release of a combination of GF (EGF, VEGF and PDGF) incorporated in 
natural polymers to improve both collagen content and remodeling rates [33].

The assessment of the histological state of the healing is essential in clinical practice for postoperative management. Epidural fibrosis is probably inevitable after any surgery that involves manipulation of the epidural space. Based on preclinical and clinical research, this epidural scarring may be the causative to persistent pain as a consequence to nerve roots compression and might reduce the ability of the spinal structures to cope with degenerative changes [8] [9]. Subsequent perineural fibrosis may interfere with cerebrospinal fluid mediated nutrition, resulting in hypersensitivity of nerve roots [7]. New studies would be proposed to prevent epidural fibrosis by avoiding primary factors rather than applying particular drugs or materials [10].

Nanofiber scaffolds produced by electrospinning mimic the 3D fibrous structure of native ECM and offer the ability to incorporate biological factors that promote regeneration [34] [35]. PA-6/SOMA scaffolds have the permeable required background provides the necessary humidity and probably offers the sustained release of the PDGF-BB, which might explain the better support to collagen replacement by the PDGF-BB-loaded PA-6/SOMA system in this study. An optimal system for biomolecule delivery has to meet some requirements, such as non-cytotoxic, mechanical strength and elasticity, and a degradation rate that supports initial tissue formation, but does not hinder maturation of the regenerated tissue due to delayed resorption.

Despite the importance of collagen deposition for repair, practical results of PRP and GF effects on healing are the subject of studies. Two systematic reviews showed that outcomes of articles was a clinical variable; for example, complete healing, bone callus formation or amount of bleeding, without examining the reasons why these results occurred [36] [37]. Despite low-level evidence animal studies and in vitro experiments describing a positive modulating effect of healing, the literature does not allow establishing the real impact of PRP or GF on cell repair phases, collagen formation or extracellular matrix deposition [36] [38] [39] [40]. Although the parameters of most scoring systems are based on basic knowledge of the healing, the parameters chosen in this experimental model focus on the scientific question underlying the hypothesis and the pathogenesis of wound healing. The set of experiments that compose this work considered the deposition of collagen a beneficial factor in the healing process but did not correlate orientation and organization of collagen fibers nor did it measure considerable patterns of tensile strength.

\section{Conclusion}

The association of PDGF-BB (platelet-derived growth factor-BB) with PA-6/SOMA (Polyamide-6 chemically modified with maleinized soybean oil) emerges as an alternative for topical application to unfavorable anatomical sites. This work highlights the potential of biomolecules to improve the wound remodeling phase through the modulation of type III and type I collagen deposition. The protec- 
tive action of the carrier matrices was pivotal to sustain the activity of the biomolecules in the wound site. In vivo histological studies in spine epidural fibrosis are in progress to further investigate the role of PDGF-BB and PRP (Platelet-rich plasma) loaded with PA-6/SOMA in all phases of the wound healing and its integration with the host tissue.

\section{Acknowledgments}

The authors thank Mrs. Caroline Nesello for her vet technical support and Mrs. Raquel Brandalise and Mrs. Simone Andriolo Gross for their technical support in animal care at the University of Caxias do Sul. We recognize and thank the expert assistance of Dr. Luciano Selistre in statistics analysis.

\section{Conflicts of Interest}

The authors declare no conflicts of interest regarding the publication of this paper.

\section{References}

[1] Velnar, T., Bailey, T. and Smrkolj, V. (2009) The Wound Healing Process: An Overview of the Cellular and Molecular Mechanisms. Journal of International Medical Research, 37, 1528-1542. https://doi.org/10.1177/147323000903700531

[2] Janis, J.E. and Harrison, B. (2014) Wound Healing: Part I. Basic Science. Plastic and Reconstructive Surgery, 138, 9s-17s. https://doi.org/10.1097/PRS.0000000000002773

[3] Gonzalez, A.C., Costa, T.F., Andrade, Z.A. and Medrado, A.R. (2016) Wound Healing-A Literature Review. Anais Brasileiros de Dermatologia, 91, 614-620. https://doi.org/10.1590/abd1806-4841.20164741

[4] Reinke, J.M. and Sorg, H.E. (2012) Wound Repair and Regeneration. European Surgical Research, 49, 35-43. https://doi.org/10.1159/000339613

[5] Diegelmann, R.F. and Evans, M.C. (2004) Wound Healing: An Overview of Acute, Fibrotic and Delayed Healing. Frontiers in Bioscience, 9, 283-289.

https://doi.org/10.2741/1184

[6] Baum, C.L. and Arpey, C.J. (2005) Normal Cutaneous Wound Healing: Clinical Correlation with Cellular and Molecular Events. Dermatologic Surgery, 31, 674-686. https://doi.org/10.1097/00042728-200506000-00011

[7] Rockey, D.C., Bell, P.D. and Hill, J.A. (2015) Fibrosis-A Common Pathway to Organ Injury and Failure. The New England Journal of Medicine, 372, 1138-1149. https://doi.org/10.1056/NEJMra1300575

[8] Sae-Jung, S., Jirarattanaphochai, K., Sumananont, C., Wittayapairoj, K. and Sukhonthamarn, K. (2015) Interrater Reliability of the Postoperative Epidural Fibrosis Classification: A Histopathologic Study in the Rat Model. Asian Spine Journal, 9, 587-594. https://doi.org/10.4184/asj.2015.9.4.587

[9] Bosscher, H.A. and Heavner, J.E. (2010) Incidence and Severity of Epidural Fibrosis after Back Surgery: An Endoscopic Study. Pain Practice, 10, 18-24. https://doi.org/10.1111/j.1533-2500.2009.00311.x

[10] Erdogan, H., Kelten, B., Tuncdemir, M., Erturkuner, S.P., Uzun, H. and Karaoglan, A. (2016) Hemostasis vs. Epidural Fibrosis? A Comparative Study on an Experi- 
mental Rat Model of Laminectomy. Neurologia i Neurochirurgia Polska, 50, 323-330. https://doi.org/10.1016/j.pjnns.2016.05.002

[11] Anitua, E., Andia, I., Ardanza, B., Nurden, P. and Nurden, A.T. (2004) Autologous Platelets as a Source of Proteins for Healing and Tissue Regeneration. Thrombosis and Haemostasis, 91, 4-15. https://doi.org/10.1160/TH03-07-0440

[12] Nurden, A.T., Nurden, P., Sanchez, M., Andia, I. and Anitua, E. (2008) Platelets and Wound Healing. Frontiers in Bioscience, 13, 3532-3548. https://doi.org/10.2741/2947

[13] Alsousou, J., Thompson, M., Hulley, P., Noble, A. and Willett, K. (2009) The Biology of Platelet-Rich Plasma and Its Application in Trauma and Orthopaedic Surgery: A Review of the Literature. The Journal of Bone and Joint Surgery. British Volume, 91, 987-996. https://doi.org/10.1302/0301-620X.91B8.22546

[14] Maitz, M.F. (2015) Applications of Synthetic Polymers in Clinical Medicine. Biosurface and Biotribology, 1, 161-176. https://doi.org/10.1016/j.bsbt.2015.08.002

[15] Ernzen, J.R., Bondan, F., Luvison, C., Wanke, C.H., Martins, J.D.N. and Fiorio, R. (2016) Structure and Properties Relationship of Melt Reacted Polyamide 6/Malenized Soybean Oil. Journal of Applied Polymer Science, 133, 10. https://doi.org/10.1002/app.43050

[16] Dias, F.T.G., Nicoletti, N.F., Marinowic, D.R., Ingracio, A., Catafesta, J., Da Costa, J.C., Falavigna, A. and Bianchi, O. (2017) Polyamide-6 Biomaterial as a Modulating Agent of Tissue Healing. Annals of the 14th Brazilian Congress of Polymers, Águas de Lindóia, 22-26 October 2017, 1311-1317.

[17] Peletti-Figueiró, M., Silveira de Aguiar, I., Paesi, S., Machado, D.C., Echeverrigaray, S., Roesch-Ely, M., Falavigna, A. and Henriques, J.A.P. (2017) Histological Markers of Degeneration and Regeneration of the Human Intervertebral Disk. Coluna/ Columna, 16, 43. https://doi.org/10.1590/s1808-185120171601170833

[18] Bonvallet, P.P., Schultz, M.J., Mitchell, E.H., Bain, J.L., Culpepper, B.K., Thomas, S.J. and Bellis, S.L. (2015) Microporous Dermal-Mimetic Electrospun Scaffolds Pre-Seeded with Fibroblasts Promote Tissue Regeneration in Full-Thickness Skin Wounds. PLoS ONE, 10, e0122359. https://doi.org/10.1371/journal.pone.0122359

[19] Kurpinski, K.T., Stephenson, J.T., Janairo, R.R., Lee, H. and Li, S. (2010) The Effect of Fiber Alignment and Heparin Coating on Cell Infiltration into Nanofibrous PLLA Scaffolds. Biomaterials, 31, 3536-3542. https://doi.org/10.1016/j.biomaterials.2010.01.062

[20] Zonari, A., Martins, T.M., Paula, A.C., Boeloni, J.N., Novikoff, S., Marques, A.P., Correlo, V.M., Reis, R.L. and Goes, A.M. (2015) Polyhydroxybutyrate-co-hydroxyvalerate Structures Loaded with Adipose Cells Promete Skin Healing with Reduced Scarring. Acta Biomaterialia, 17, 170-181.

https://doi.org/10.1016/j.actbio.2015.01.043

[21] Bonvallet, P.P., Culpepper, B.K., Bain, J.L., Schultz, M.J., Thomas, S.J. and Bellis, S.L. (2014) Microporous Dermal-Like Electrospun Scaffolds Promote Accelerated Skin Regereneration. Tissue Engineering Part A, 20, 2434-2445.

https://doi.org/10.1089/ten.tea.2013.0645

[22] Mahjour, S.B., Fu, X., Yang, X., Fong, J., Sefat, F. and Wang, H. (2015) Rapid Creation of Skin Substitutes from Human Skin Cells and Biomimetic Nanofibers for Acute Full-Thickness Wound Repair. Burns, 41, 1764-1774.

https://doi.org/10.1016/j.burns.2015.06.011

[23] Yeh, C.J., Chen, C.C., Leu, Y.L., Lin, M.W., Chiu, M.M. and Wang, S.H. (2017) The Effects of Artocarpin on Wound Healing: In Vitro and in Vivo Studies. Scientific 
Reports, 7, Article No. 15599. https://doi.org/10.1038/s41598-017-15876-7

[24] Mori, H.M., Kawanami, H., Kawahata, H. and Aoki, M. (2016) Wound Healing Potential of Lavender Oil by Acceleration of Granulation and Wound Contraction through Induction of TGF- $\beta$ in a Rat Model. BMC Complementary and Alternative Medicine, 16, 114. https://doi.org/10.1186/s12906-016-1128-7

[25] Fang, R.C. and Galiano, R.D. (2008) A Review of Becaplermin Gel in the Treatment of Diabetic Neuropathic Foot Ulcers. Biologics, 2, 1-12. https://doi.org/10.2147/BTT.S1338

[26] Marx, R.E. (2001) Platelet-Rich Plasma (PRP): What Is PRP and What Is Not PRP? Implant Dentistry, 10, 225-228. https://doi.org/10.1097/00008505-200110000-00002

[27] Dhurat, R. and Sukesh, M. (2014) Principles and Methods of Preparation of Platelet-Rich Plasma: A Review and Author's Perspective. Journal of Cutaneous and Aesthetic Surgery, 7, 189-197. https://doi.org/10.4103/0974-2077.150734

[28] Mensik, I., Lamme, E.N. and Riesle, J. (2002) Effectiveness and Safety of the PEGT/PBT Copolymer Scaffold as Dermal Substitute in Scar Reconstruction Wounds (Feasibility Trial). Cell and Tissue Banking, 3, 245-253.

https://doi.org/10.1023/A:1024674325253

[29] Martin, P., Teodoro, W.R., Velosa, A.P., Morais, J., Carrasco, S. and Christmann, R.B. (2012) Abnormal Collagen V Deposition in Dermis Correlates with Skin Thickening and Disease Activity in Systemic Sclerosis. Autoimmunity Reviews, 11, 827-835. https://doi.org/10.1016/j.autrev.2012.02.017

[30] Kuppan, P., Vasanthan, K.S., Sundaramurthi, D., Krishnan, U.M. and Sethuraman, S. (2011) Development of Poly(3-hydroxybutyrate-co-3-hydroxyvalerate) Fibers for Skin Tissue Engineering: Effects of Topography, Mechanical, and Chemical Stimuli. Biomacromolecules, 12, 3156-3165. https://doi.org/10.1021/bm200618w

[31] Barnes, M.J., Morton, L.F., Bennett, R.C., Bailey, A. and Sims, T.J. (1976) Presence of Type III Collagen in Guinea-Pig Dermal Scar. Biochemical Journal, 157, 263-266. https://doi.org/10.1042/bj1570263

[32] Al-Qattan, M.M., Abd-Elwahed, M.M., Hawary, K., Arafah, M.M. and Shier, M.K. (2015) Myofibroblast Expression in Skin Wounds Is Enhanced by Collagen III Supression. BioMed Research International, 2015, Article ID: 958695. https://doi.org/10.1155/2015/958695

[33] Lai, H.J., Kuan, C.H., Wu, H.C., Tsai, J.C., Chen, T.M., Hsieh, D.J. and Wang, T.W. (2014) Tailored Design of Electrospun Composite Nanofibers with Staged Release of Multiple Angiogenic Growth Factors for Chronic Wound Healing. Acta Biomaterialia, 10, 4156-4166. https://doi.org/10.1016/j.actbio.2014.05.001

[34] Vasita, R., Shanmugam, I.K. and Katt, D.S. (2008) Improved Biomaterials for Tissue Engineering Applications: Surface Modification of Plymers. Current Topics in $\mathrm{Me}$ dicinal Chemistry, 8, 341-353. https://doi.org/10.2174/156802608783790893

[35] Ravichandran, R., Sundarrajan, S., Venugopal, J.R., Mukherjee, S. and Ramakrishna, S. (2012) Advances in Polymeric Systems for Tissue Engineering and Biomedical Applications. Macromolecular Bioscience, 12, 286-311. https://doi.org/10.1002/mabi.201100325

[36] Smyth, N.A., Murawski, C.D., Fortier, L.A., Cole, B.J. and Kennedy, J.G. (2013) Platelet-Rich Plasma in the Pathologic Processes of Cartilage: Review of Basic Science Evidence. Arthroscopy, 29, 1399-1409.

https://doi.org/10.1016/j.arthro.2013.03.004

[37] Anitua, E., Sanchez, M. and Orive, G. (2010) Potential of Endogenous Regenerative 
Technology for in Situ Regenerative Medicine. Advanced Drug Delivery Reviews, 62, 741-752. https://doi.org/10.1016/j.addr.2010.01.001

[38] Anitua, E., Tejero, R., Zalduendo, M.M. and Orive, G. (2013) Plasma Rich in Growth Factors Promotes Bone Tissue Regeneration by Stimulating Proliferation, Migration, and Autocrine Secretion in Primary Human Osteoblasts. Journal of Periodontology, 84, 1180-1190. https://doi.org/10.1902/jop.2012.120292

[39] Anitua, E., Pino, A. and Orive, G. (2016) Plasma Rich in Growth Factors Promotes Dermal Fibroblast Proliferation, Migration and Biosynthetic Activity. Journal of Wound Care, 25, 680-687. https://doi.org/10.12968/jowc.2016.25.11.680

[40] Sanchez, M., Anitua, E., Delgado, D., Sanchez, P., Prado, R. and Orive, G. (2017) Platelet-Rich Plasma, a Source of Autologous Growth Factors and Biomimetic Scaffold for Peripheral Nerve Regeneration. Expert Opinion on Biological Therapy, 17, 197-212. https://doi.org/10.1080/14712598.2017.1259409 


\section{Suplementary Figures}

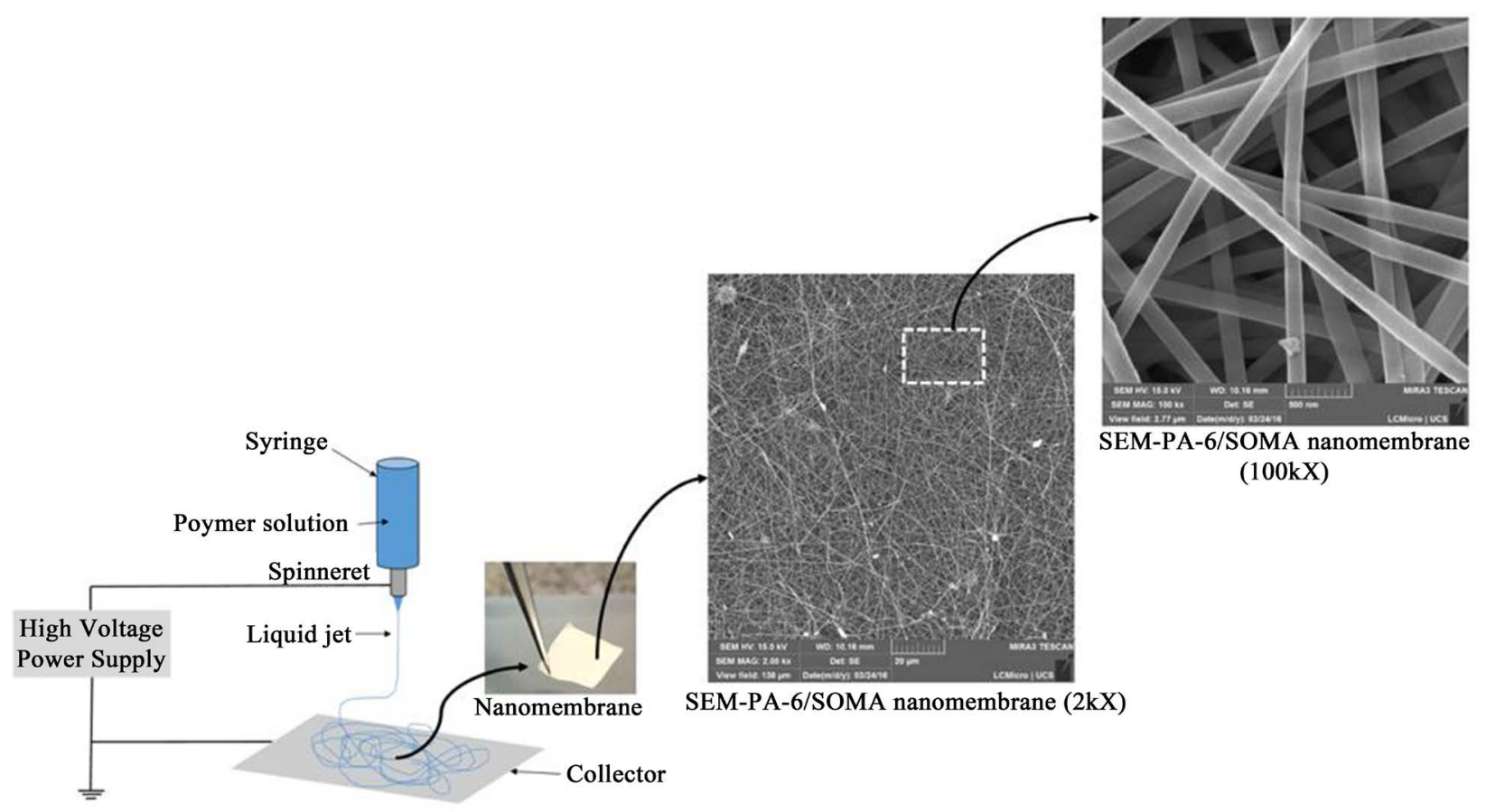

Figure S1. Scheme for nanomembrane production and SEM analysis.

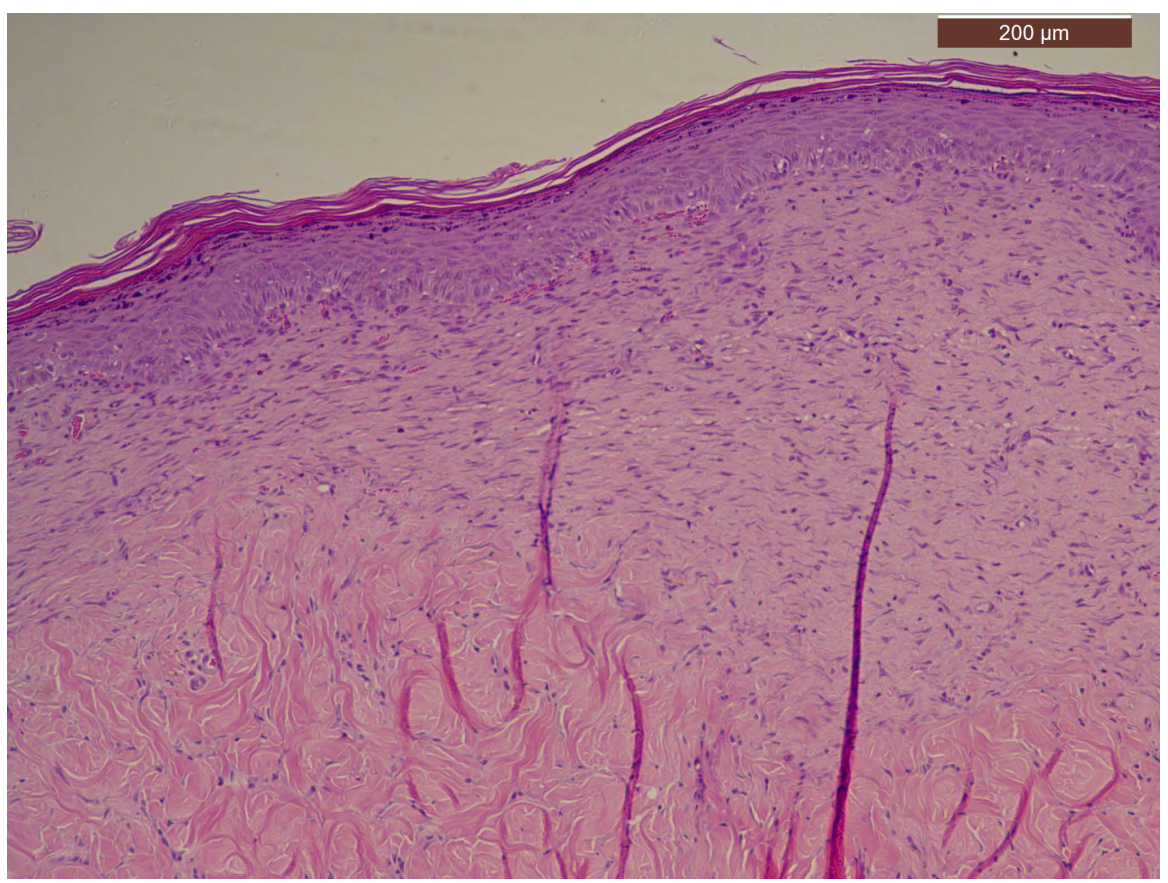

Figure S2. Demonstrative image of Hematoxylin and Eosin (H\&E) stained in the studied samples $(200 \times$ objective). 